\title{
Hepatite B: prevalência de marcadores sorológicos em profissionais de enfermagem de emergência
}

\author{
Hepatitis B: prevalence of serological markers in emergency nursing professionals \\ Hepatitis B: prevalencia de marcadores serológicos en profesionales de enfermería \\ de emergencia
}

\author{
Telma Maria Evangelista de Araújo'; Nayra da Costa e Silva ${ }^{I I}$
}

\begin{abstract}
RESUMO: Estudo quantitativo, descritivo, transversal com objetivo de avaliar a prevalência dos marcadores sorológicos para Hepatite B em profissionais de enfermagem dos serviços de emergência de Teresina/Piauí. Realizado de março a maio de 2010, com 317 profissionais de enfermagem de cinco hospitais públicos. Predominaram mulheres (94,3\%), casados $(54,6 \%)$, técnicos de enfermagem (59,9\%) e tempo médio de profissão de 1 a 20 anos (55,5\%). Não ocorreu positividade nos marcadores $\mathrm{HBsAg}$ e HBc total na população investigada. O Anti-HBs foi encontrado em maior evidência nos técnicos $(32,6 \%)$ e auxiliares de enfermagem $(31,7 \%)$. Houve associação estatisticamente significativa entre o Anti-HBs e as variáveis, número de doses de vacina e tempo de profissão $(p=0,03)$. O controle sorológico do Vírus da Hepatite B é importante para o controle da progressão da doença em profissionais de enfermagem, porém a ampliação do acesso a essas medidas constitui um grande desafio.
\end{abstract}

Palavras-Chave: Hepatite B; enfermagem; prevenção; saúde do trabalhador.

ABSTRACT: This study was quantitative, descriptive and transversal and aimed at evaluating the prevalence of serological markers for Hepatitis B in nursing professionals from the emergency services in Teresina, Piauí, Brazil. It was held from March to May, 2010, with 317 nurses from five public hospitals. Females (94.3\%), married (54.6\%), nursing technicians (59.9\%), and the average employment length of $1-20$ years $(55.5 \%)$ predominated. There was no positivity for $\mathrm{HBsAg}$ and $\mathrm{HBc}$ total in the population investigated. Anti-HBs was found in greater evidence in nursing technicians and assistants $(32.6 \%)$ and $(31.7 \%)$, respectively. There was statistically significant association between anti-HBs and variables, number of vaccine doses and length of employment $(p=0.03)$. The serological control of the Hepatitis B virus is important for the control of disease progression in nursing professionals, but the expansion of access to these measures is a major challenge.

Keywords: Hepatitis B; nursing; prevention; occupational health.

RESUMEN: Estudio cuantitativo, descriptivo y transversal para evaluar la prevalencia de marcadores serológicos de la Hepatitis B en los profesionales de enfermeira de los servicios de emergencia en Teresina/Piauí - Brasil. Realizado de marzo a mayo de 2010, con 317 profesionales de enfermería de cinco hospitales públicos. Predominaron mujeres (94,3\%), casados (54,6\%), tecnicos de enfermería (59,9\%) y la duración del empleo de $1-20$ años $(55,5 \%)$. No hubo positividad para HBsAg y HBc total en la población investigada. Anti-HBs se encontró en mayor evidencia en los técnicos y auxiliares de enfermería $(32,6 \%)$ y $(31,7 \%)$. Hubo asociación estadísticamente significativa entre los anti-HBs y las variables, número de dosis de vacuna y la duración del empleo $(p=0,03)$. El control serológico de la Hepatitis B es importante para el control de la progresión de la enfermedad en los profesionales de enfermería, pero la expansión del acceso a estas medidas es un gran desafío.

Palabras Clave: Hepatitis B; enfermería; prevención; salud del trabajador.

\section{INTRODUÇÃO}

O impacto da Hepatite B no cenário mundial tem sido representado pela elevada taxa morbidade e mortalidade. Estimativas apontam que 360 milhões de pessoas estão cronicamente infectados e que 600.000 pessoas morram por ano devido às consequências agudas ou crônicas da doença. A prevalência da infecção crônica pelo Vírus de Hepatite B (VHB) é altamente variável, sendo de $0,1 \%$ nos Estados Unidos para 20 $30 \%$ em algumas nações insulares do Pacífico ${ }^{1}$.
No Brasil, segundo o boletim epidemiológico do ano de 2012, a Região Sudeste concentrou 36,6\% dos casos, seguida do Sul, com 31,6\% das notificações, e a média nacional para casos de Hepatite B no Brasil passou de 0,3\%, em 1999, para 6,9\%, em 2010².

Além da prevalência da doença na população geral, os grupos de risco para a Hepatite B devem ser observados com bastante cautela. Estudos epidemiológicos em grupos específicos coadunam com o

'Doutora em Enfermagem. Professora Adjunta da Graduação e do Programa de Mestrado em Enfermagem da Universidade Federal do Piauí. Diretora da Vigilância e da Atenção em Saúde do Estado do Piauí. Teresina, Piauí, Brasil. E-mail: telmaevangelista@gmail.com

IIMestre em Enfermagem. Professora Adjunta do Centro Tecnológico de Teresina/Universidade Federal do Piauí. Teresina, Piauí, Brasil. E-mail: nayralygil@hotmail.com. 
interesse deste artigo, onde a investigação dos perfis sorológicos de grupos vulneráveis, bem como os comportamentos de risco, podem levar à compreensão dos fatores que induzem à exposição da doença ${ }^{3,4}$.

Dados da Organização Internacional do Trabalho identificam, anualmente, 160 milhões de doenças ocupacionais e 250 milhões de acidentes de trabalho ${ }^{5}$. Destaca-se que a Hepatite B ainda é a doença de maior prevalência entre os trabalhadores da área de saúde e tem contribuído para os elevados índices de doenças ocupacionais, constituindo, portanto, um grave problema de saúde pública ${ }^{1}$.

Somente com o advento da AIDS, na década de 1980, houve uma preocupação pelas instituições que regulamentam a saúde do trabalhador, em estabelecer medidas de precaução padrão e normas para as atividades no ambiente laboral dos profissionais de saúde. Pesquisas apontam que VHB circula em altas concentrações no sangue e em títulos baixos nos outros fluidos orgânicos, sendo aproximadamente 100 vezes mais infectante do que o HIV e 10 vezes mais do que o Vírus de Hepatite C (VHC) ${ }^{6}$.

Dentro dessa problemática, a situação sorológica dos profissionais que atuam nos serviços de saúde é primordial para prevenção e controle do VHB no ambiente laboral. Assim, o objetivo desse estudo foi o de avaliar a prevalência dos marcadores sorológicos para Hepatite B nos profissionais de enfermagem que atuam nos serviços de urgência e emergência.

\section{REVISÃo DE LITERATURA}

Estruturalmente, o VHB pertence à família Hepadnaviridae, possui 3.200 pares de base, tem sua forma molecular constituída de um núcleo de DNA de cadeia dupla e possui dois antígenos importantes para determinação da presença do vírus e do curso da doença, que são os antígenos centrais e o de superfície?

O homem é considerado o único reservatório de importância epidemiológica e pode transmitir a doença de várias formas: via parenteral (transfusional, antes da política de triagem em bancos de sangue; compartilhamento de agulhas, seringas ou outros equipamentos contendo sangue contaminado; procedimentos médicos/odontológicos com sangue contaminado, realização de tatuagens e colocação de piercings); sexual (em relações desprotegidas); por meio de solução de continuidade (pele e mucosa). Há evidências que sugerem a possibilidade de transmissão por compartilhamento de instrumentos de manicure, escovas de dente, lâminas de barbear ou de depilar, canudo de cocaína, cachimbo de crack, entre outros ${ }^{1}$.

De forma geral, o diagnóstico diferencial da Hepatite B só é possível por meio da detecção dos marcadores sorológicos. São marcadores de triagem para a hepatite B: HBsAg (o primeiro marcador a surgir após a infecção pelo VHB); Anti-HBc (é um marcador que indica contato prévio com o vírus); Anti-HBc IgM (é um marcador de infecção recente, portanto, confirma o diagnóstico de hepatite $\mathrm{B}$ aguda); Anti-HBs (indica imunidade contra o VHB); HBeAg (é indicativo de replicação viral e, portanto, de alta infectividade) e Anti-HBe (marcador de bom prognóstico na hepatite aguda pelo $\mathrm{HBV})^{8}$.

O conhecimento dos marcadores sorológicos para Hepatite B é de fundamental importância, uma vez que a situação sorológica dos indivíduos expostos pode determinar o curso natural da doença e são essenciais para o desenvolvimento de estratégias voltadas para a saúde do trabalhador ${ }^{9}$. Estudo sobre a exposição ocupacional a fluidos corporais entre trabalhadores de saúde na Geórgia reforçam a preocupação com a progressão da Hepatite B no ambiente hospitalar quanto á vulnerabilidade do profissional de saúde á exposição do VHB, onde os resultados dessas pesquisas comprovam a presença de marcadores sorológicos para Hepatite B nesses profissionais ${ }^{10}$.

\section{Metodologia}

Trata-se de uma pesquisa de natureza quantitativa, descritiva e transversal, realizada por meio de um inquérito soro-epidemiológico efetivado em cinco Hospitais da Fundação Municipal de Saúde (FMS), que prestam serviços de urgência e emergência no atendimento à população, bem como, serviços ambulatoriais, de maternidade e de apoio à estratégia saúde da família.

A população da pesquisa foi composta pelo universo de profissionais de enfermagem dos serviços de urgência e emergência $(n=360)$, distribuídos em três categorias: enfermeiros, técnicos e auxiliares. $\mathrm{O}$ critério de exclusão foi o não consentimento na participação no estudo, encontrar-se de férias ou licença no período da coleta.

A pesquisa foi realizada em consonância com preceitos éticos que regulamentam os estudos envolvendo seres humanos, sendo aprovada pela Comissão de Ética em Pesquisa da Universidade Federal do Piauí-UFPI (CAAE nº 0163. 0.045.000-08).

A coleta de dados foi realizada no período de março a maio de 2010, por meio de duas etapas: primeiramente, realizou-se a entrevista através de formulário composto de perguntas relacionadas às características socioepidemiológicas e situação sorológica dos participantes da pesquisa e na segunda etapa, foram realizados os testes sorológicos, conforme orientação do Laboratório Central do Piauí (LACEN). Foram pesquisados os marcadores sorológicos, com fins de diagnóstico da Hepatite B (HBsAg, Anti-HBs, HBc Total), nas salas de coleta de cada hospital, nos períodos matutino e noturno, respeitando a escala de plantão e as atividades laborais dos sujeitos 
do estudo. Os testes utilizados foram: HBsAg (Vikia®), HBc total (IEA WELL), Anti-HBs (IEMA WELL). Este estudo tomou como definição de casos: imune por infecção (indivíduos com Anti-HBc e Anti-HBs positivos), imune pela vacina (indivíduos com Anti-HBs positivo) e sem proteção (indivíduos com Anti-HBc e Anti-HBs negativo).

As variáveis levantadas foram: faixa etária, sexo, situação conjugal, categoria profissional, tempo de profissão, marcadores sorológicos, tempo de vacinado e número de doses de vacinas recebidas contra a Hepatite B.

Para análise dos dados foi construído um banco de dados, o qual foi explorado por meio de técnicas univariadas e bivariadas utilizando-se o software Statiscal Package for the Social Science (SPSS) 17.0. A análise univariada foi realizada por meio do cálculo de estatísticas descritivas das variáveis relacionadas à descrição da amostra e da realização da sorologia da Hepatite B anterior à pesquisa. Para a realização das análises bivariadas, calculou-se o Qui-quadrado de Pearson $\left(\mathcal{X}^{2}\right)$, objetivando identificar possíveis associações entre as variáveis dependentes (resultados do Anti-HBs e HBc Total) com cada variável independente. A hipótese nula foi rejeitada quando o valor de $p$ foi menor ou igual a 0,05. Os achados mais significativos foram representados em tabelas e posteriormente, analisados à luz da produção científica dos autores da área temática.

A população planejada para participar do estudo foi de 360 profissionais. Entretanto, 39 encontravamse afastados por férias ou licença no período da coleta dos dados, sendo retirados da pesquisa pelo critério de exclusão previsto no planejamento. Entre os 321, quatro se recusaram a participar da pesquisa, totalizando 317 profissionais estudados.

\section{Resultados e Discussão}

Dos 317 profissionais que participaram do estudo, 121 (38,2\%) estavam na faixa etária de 41 a 50 anos, predominou o sexo feminino, com 229(94,3\%) e, no que diz respeito à situação conjugal, 173(54,6\%) eram casados. Na variável categoria profissional, os técnicos de enfermagem representaram 59,9\% da amostra e o tempo médio de profissão foi de 1 a 20 anos. Na caracterização dos sujeitos do estudo, estatísticas mostram que as mulheres representam 73,0\% dos empregos na área da saúde. A intensificação da participação feminina, no trabalho de enfermagem, iniciou na década de 70 , com predominância ainda constante nesse setor ${ }^{11,12}$.

Os aspectos sociodemográficos dos participantes do estudo estão apresentados na Tabela 1.

Os profissionais de enfermagem, independente do nível de formação, estão vulneráveis a con-
TABELA 1: Descrição da população do estudo. Teresina-PI, $2010(\mathrm{~N}=317)$.

\begin{tabular}{lc}
\hline \multicolumn{1}{c}{ Variáveis } & $\mathrm{f}(\%)$ \\
\hline Faixa etária (em anos) & $112(35,3)$ \\
20 a 40 & $121(38,2)$ \\
41 a 50 & $84(26,5)$ \\
51 a 68 & \\
Sexo & $229(94,3)$ \\
$\quad$ Feminino & $18(5,7)$ \\
Masculino & \\
Situação conjugal & $173(54,6)$ \\
$\quad$ Casado & $101(31,9)$ \\
$\quad$ Solteiro & $25(7,9)$ \\
$\quad$ Separado & $18(5,7)$ \\
Viúvo & \\
Categoria profissional & $83(26,2)$ \\
$\quad$ Auxiliar de enfermagem & $44(13,9)$ \\
Enfermeiro & $190(59,9)$ \\
Técnico em enfermagem & \\
Tempo de profissão (em anos) & $176(55,5)$ \\
1 a 20 & $141(44,5)$ \\
21 a 42
\end{tabular}

taminação pelos mais diversos microorganismos no ambiente hospitalar. Quando comparados as outras categorias profissionais, pesquisas são unânimes ao afirmarem que a equipe de enfermagem sofre acidente com maior freqüência no ambiente hospitalar ${ }^{13-15}$. Esse fato assemelha-se ao desta pesquisa, em que um expressivo percentual de profissionais de enfermagem relatou ter sofrido algum tipo de acidente com material biológico $(47,9 \%)$, sendo que o acidente com agulha foi o de maior proporção (36,9\%).

Uma das medidas profiláticas no controle da progressão da Hepatite B é o controle sorológico. Os profissionais de saúde ao iniciarem as práticas hospitalares devem seguir as condutas que seguem: imunização prévia com a vacina Hepatite $B$, controle sorológico do Anti-HBs e para os que ainda não completaram o esquema contra hepatite é necessário pesquisar a presença do HBsAg. Frente à condição de não reator devem completar o esquema e, após um intervalo de um a seis meses, realizar o controle sorológico ${ }^{16,17}$.

Quando investigados sobre a realização de sorologia em algum outro momento anterior ao da pesquisa, a categoria profissional dos auxiliares de enfermagem foi a que se sobressaiu com relação às demais $(91,6 \%)$, afirmando nunca ter realizado este exame.

Quanto aos resultados da sorologia para Hepatite B obtidos neste estudo, pode-se verificar que nenhum profissional apresentou positividade para o $\mathrm{HBsAg}$ e somente 9,2\% foram reagentes ao Anti-HBc total, conforme expõe a Tabela 2. Isso mostra que, em algum 
momento da vida, estes participantes tiveram contato com o Vírus da Hepatite B, pois este marcador dá positivo na fase entre o desaparecimento dos antígenos e o aparecimento dos anticorpos. Pesquisas de marcadores sorológicos realizados com profissionais de saúde encontraram resultados semelhantes - 9,4\% e 8,1\%, respectivamente, quanto ao resultado do Anti-HBc total ${ }^{18,19}$. Todavia, alguns estudos internacionais, em países de alta endemicidade para o VHB, mostram a presença do marcador HBsAg em profissionais de saúde ${ }^{20,21}$.

Infere-se que a não existência de casos positivos de HBsAg na população investigada pode ser justificada pela amostra insuficiente de indivíduos portadores, pois a prevalência global do marcador $\mathrm{HBsAg}$, referente ao conjunto das capitais do Brasil e o Distrito Federal é de 0,37\%, classificado como de baixa endemicidade ${ }^{22}$. Porém, mesmo diante da baixa prevalência, os fatores de risco da população investigada, frente à exposição aos acidentes biológicos e ao esquema vacinal incompleto ou ausente, ainda constituem-se em lacunas que interferem no controle da doença.

Outro importante marcador de prevalência da Hepatite B, realizado nesta pesquisa, foi o Anti-HBs. Ele surge após o desaparecimento do HBsAg e indica que o indivíduo está imune à infecção pelo vírus ou pela imunização completa. Neste estudo, um percentual expressivo de pesquisados $(30,3 \%)$ não foi reagente a esse marcador.

Achados semelhantes foram relatados em uma pesquisa realizada em um hospital privado, onde foram realizadas 1.115 sorologias para o Anti-HBs em profissionais de saúde de risco baixo ou alto para a ocorrência de acidentes ocupacionais ${ }^{23}$. Dos exames realizados, 729 foram reagentes e 386 não reagentes, caracterizando um percentual de soropositividade de $65,4 \%$. Entretanto, estudo divergente deste apontou que apenas $2,4 \%$ dos pesquisados apresentaram resultados positivos para o Anti- $\mathrm{HBs}^{24}$.

Nos resultados dos marcadores sorológicos, foi observado que 24 participantes, distribuidos nas três categorias profissionais, apresentaram simultaneamente positividade para os marcadores $\mathrm{HBc}$-total e Anti-HBs, demonstrando que eles estão imunes por infecção. A imunidade adquirida naturalmente é estabelecida pela presença concomitante do Anti-HBs e Anti-HBc IgG ou total. Eventualmente, o Anti-HBc pode ser o único indicador da imunidade natural detectável sorologicamente, pois com o tempo, o nível de Anti-HBs considerado protetor $(10 \mathrm{~m} \mathrm{UI} / \mathrm{ml})$ pode tornar-se indetectável. A ocorrência do Anti-HBs, como marcador isolado de imunidade contra o HBV, adquirida naturalmente, é possível, embora seja muito pouco frequente. Assim, são susceptíveis à patologia pessoas com perfil sorológico $\mathrm{HBsAg}$, Anti-HBc e Anti-HBs negativos concomitantemente ${ }^{25}$.

Neste estudo, houve associação estatística significativa entre o Anti-HBs com o número de doses da vacina contra Hepatite $B(p=0,03)$, demonstrada na Tabela 3. Esta vacina oferece uma boa proteção quando administrada em doses e especialmente com intervalos adequados. Entretanto, nas pessoas com idade avançada, a resposta é menos efetiva ${ }^{26}$. Pesquisa semelhante coaduna com esta quando associa os valores do Anti-HBs ao número de doses da vacina entre profissionais de saúde ${ }^{24}$.

A duração da imunidade após a vacinação contra a Hepatite B tem sido objeto de muitas pesquisas. Outro estudo realizado demonstrou o declínio dos títulos de Anti-HBs algum tempo após a vacinação.

TABELA 2: Soroprevalência da Hepatite B na população do estudo. Teresina-PI, 2010. $(\mathrm{N}=309)$

\begin{tabular}{|c|c|c|c|}
\hline \multirow{2}{*}{ Marcadores } & \multicolumn{3}{|c|}{ Categoria Profissional } \\
\hline & $\begin{array}{c}\text { Auxiliar de } \\
\text { enfermagem } \\
f(\%)\end{array}$ & $\begin{array}{c}\text { Técnico de } \\
\text { enfermagem } \\
f(\%)\end{array}$ & $\begin{array}{l}\text { Enfermeiro } \\
\qquad f(\%)\end{array}$ \\
\hline \multicolumn{4}{|l|}{$\mathrm{HBsAg}$} \\
\hline Não reagente & $82(100)$ & $187(100)$ & $40(100)$ \\
\hline \multicolumn{4}{|l|}{ HBc -Total } \\
\hline Não reagente & $75(91,5)$ & $170(90,9)$ & $35(87,5)$ \\
\hline Reagente & $7(8,5)$ & $17(9,1)$ & $5(12,5)$ \\
\hline \multicolumn{4}{|l|}{ Anti-HBs } \\
\hline Não reagente & $26(31,7)$ & $61(32,6)$ & $8(20,0)$ \\
\hline Reagente & $56(68,3)$ & $126(67,4)$ & $32(80,0)$ \\
\hline $\begin{array}{l}\text { HBc Total e Anti-Hbs } \\
\text { Reagentes }\end{array}$ & $7(29,2)$ & $13(54,1)$ & $4(16,7)$ \\
\hline
\end{tabular}


TABELA 3: Associação do Anti-HBs com as variáveis: tempo de vacinado, faixa etária, categoria profissional, número de doses de vacina contra a hepatite B. Teresina-PI, 2010.

\begin{tabular}{|c|c|c|c|c|}
\hline \multirow[b]{2}{*}{ Variáveis } & \multicolumn{4}{|c|}{ AntiHBs } \\
\hline & $\begin{array}{c}\text { Não reagente } \\
f(\%)\end{array}$ & $\begin{array}{c}\text { Reagente } \\
f(\%)\end{array}$ & $\mathrm{x}^{2}$ & p valor \\
\hline Tempo de vacinado/anos $(n=289)$ & & & 0,008 & 0,93 \\
\hline Mais de 1 ano & $73(29,3)$ & $176(70,7)$ & & \\
\hline Menos de 1 ano & $12(30,0)$ & $28(70,0)$ & & \\
\hline Faixa etária $(n=309)$ & & & 1,523 & 0,21 \\
\hline 40 anos e mais & $66(35,7)$ & $136(67,3)$ & & \\
\hline 20 a 40 anos & $29(27,1)$ & $78(72,9)$ & & \\
\hline Categoria profissional $(n=309)$ & & & 2,513 & 0,28 \\
\hline Auxiliar de Enfermagem & $26(31,7)$ & $56(68,3)$ & & \\
\hline Técnico em enfermagem & $61(32,6)$ & $126(67,4)$ & & \\
\hline Enfermeiro & $8(20,0)$ & $32(80,0)$ & & \\
\hline$N^{\circ}$ de doses de vacina $(n=205)$ & & & 10,241 & 0,03 \\
\hline 1 dose & $6(16,2)$ & $31(83,8)$ & & \\
\hline 2 doses & $24(38,7)$ & $38(61,3)$ & & \\
\hline 3 doses & $44(27,7)$ & $115(72,3)$ & & \\
\hline
\end{tabular}

Entre os adultos, os níveis de Anti-HBs declinam para menos que $10 \mathrm{~m} \mathrm{UI} / \mathrm{ml} \mathrm{em} 7 \%$ a $50 \%$ dos vacinados, 5 anos após a vacinação; e em 30\% a 60\% dos vacinados, entre 9 a 11 anos após a vacinação ${ }^{25}$.

A memória imunológica tem sido evidenciada pelo rápido aumento nos anticorpos Anti-HBs, seguido de uma dose de reforço administrada vários anos após a série primária, fato importante para a manutenção da proteção, mesmo quando os níveis séricos dos anticorpos específicos apresentam-se baixos $^{27}$. Não obstante ter sido constatado por muitos estudiosos do assunto, ainda permanece como foco de muitos debates o reforço da vacina contra Hepatite B.

A resposta imunológica depende de uma multiplicidade de fatores. $\mathrm{O}$ avançar da idade piora a resposta à vacinação devido ao decréscimo da atividade de células imunológicas T e B. O que explica o fato de que, neste estudo, $31,9 \%$ dos pesquisados com faixa etária acima de 40 não terem sido reagentes ao marcador Anti-HBs ${ }^{18}$.

Sendo a Hepatite B a doença ocupacional mais investigada nos profissionais de enfermagem, ressaltase que o conhecimento da situação sorológica desses trabalhadores constitui uma das formas de controle e prevenção da doença e essas ações devem ser priorizadas nos serviços hospitalares e não somente na ocorrência da exposição ocupacional ${ }^{28}$.

\section{CONCLUSÃo}

Evidenciou-se, no estudo, que algumas áreas específicas, como os serviços de urgência e emergência, podem favorecer as exposições ocupacionais de toda natureza, proporcionando uma maior vulnerabilidade à equipe de enfermagem.
Quanto aos marcadores investigados na pesquisa, destaca-se que embora não se tenha sido encontrado infecção atual pelo VHB, foi detectada uma parcela considerável de profissionais com títulos protetores insatisfatórios para conferir imunidade contra a doença, fato que os tornam susceptíveis a contaminações futuras, caso não busquem completar a imunização ou não adotem as medidas cabíveis.

Como limitações do estudo podemos destacar: o medo da submissão à coleta e dos resultados das sorologias por parte dos profissionais de enfermagem, o cotidiano exaustivo dos setores de urgência que muitas vezes dificultaram o andamento do estudo. Espera-se que o presente estudo possa estimular os profissionais de saúde, em especial, a equipe de enfermagem, a uma reflexão sobre a progressão da Hepatite $\mathrm{B}$, como doença ocupacional, de modo, a adotarem ações que possam minimizar os riscos no ambiente de trabalho.

\section{REFERÊNCIAS}

1. World Health Organization [Internet].Hepatitis B.2014.[citado em 15 out 2014] Disponível em: http:// www.who.int/mediacentre/factsheets/fs204/en/

2.Ministério da Saúde (Brasil), Secretaria de Vigilância em Saúde /Departamento de DST, Aids e Hepatites Virais. Boletim epidemiológico Hepatites Virais. Brasília (DF): Ministério da Saúde; 2012.

3.Lopes LPL, Teles SAT, Romão EA, Toffano SEM, Rocha DFNC, Gir E. Vacinação contra Hepatite B em indivíduos renais crônicos em tratamento hemodialítico. Rev enferm UERJ. 2014; 22: 309-13.

4.Rossi GC, Afonso PMD, Oliveira SLGO, Furlan MLS. Hepatites B e C: o conhecimento dos estudantes universitários da área da saúde. Rev enferm UERJ. 2010; 18: 38-41. 
5.Gasparini LR. Controle de infecção. São Paulo: Becton Dickinson; 2005

6.Center for Disease Control and Prevention [Internet]. Recommendations for prevention of Hepatitis B and C.2008 [cited in 2014 Jun 2014] Available in: http:// www.cdc.gov/hepatitis/HBV/TestingChronic.htm

7.Khouri M, Santos V. Hepatitis B: epidemiological, immunological, and serological considerations emphasizing mutation. Rev Hosp Clín. 2004; 59:97-100.

8.Ministério da Saúde (Brasil), Programa nacional de hepatites virais. Manual de aconselhamento em hepatites virais. Brasília (DF): Ministério da Saúde; 2005.

9.Caetano MM, Beck ST. Importância da detecção de anticorpos anti-HBc na prevenção da transmissão do vírus da Hepatite $B(\mathrm{VHB})$ em bancos de sangue. RBAC. 2006; 38: 235-7.

10.Butsashvili M, Kamkamidze G, Kajaia M, Morse DL, Triner W, DeHovitz L, McNutt LA. Occupational exposure to body fluids among health care workers in Georgia. Occup Med. 2012; 62:620-6.

11.Moraes EL. Relação gênero e raça na política pública de qualificação social e profissional. Brasília (DF): Ministério do Trabalho e Emprego; 2005.

12.Almeida MCP, Robazzi MLCC, Scochi CGS, Bueno SMV, Cassiani SHB, Saeki T, et al. Perfil da demanda dos alunos da pós-graduação scricto sensu da escola de enfermagem de Ribeirão Preto, da Universidade de São Paulo. Rev Latino-Am Enferm. 2004; 12: 153-61.

13. Ribeiro EJG, Shimizu HE. Acidentes de trabalho com trabalhadores de enfermagem. Rev Bras Enferm. 2007; 60: 535-40.

14.Balsamo AC, Felli VEA. Estudo sobre os acidentes de trabalho com exposição aos líquidos corporais humanos em trabalhadores da saúde de um hospital universitário. Rev Latino-Am Enferm. 2006; 14: 346-53.

15.Pinho DLM, Rodrigues CM, Gomes GP. Perfil dos acidentes de trabalho no Hospital Universitário de Brasília. Rev Bras Enferm. 2007; 60: 291-4

16.Araújo TME, Paz EPA, Griep RH. Cobertura vacinal dos profissionais de um curso de especialização em Saúde da Família do Piauí. Esc Anna Nery. 2006; 10: 95-100. 17.Pinheiro J, Zeitoune RCG. Hepatite B e a saúde do trabalhador de enfermagem. Esc Anna Nery.2008; 12: 258-64.
18.Ciorlia LAS, Zanetta DMT .Hepatitis B in healthcare workers: prevalence, vaccination and relation to occupational factors. Braz J Infect Dis.2005; 9: 384-9.

19.Kondili LA, Ulginaku D, Haidini M, Basho M, Chionne P, Madonna E, et al. Hepatitis B virus Infection in health care workers in Albania: a country still highly endemic for HBV Infection. 2007. [cited in 2014 Jan 01]. Available in: http://www.springerlink.com/content/15v0738329p0058x/ 20.Doddaiah V, Janakiram K, Javagal S. Seroprevalence of Hepatitis B virus and Hepatitis C virus in healthcare workers - AIMS, B G Nagara. Am J Life Sci. 2013; 1(4):145-9 21.Mosendane T, Kew MC, Osih R, Mahomed A. Nurses at risk for occupationally acquired blood-borne virus infection at a South African academic hospital. S Afr Med J. [Internet]. 2012. [cited in 2014 Nov 15] Available in: http:// www.samj.org.za/index. php/samj/article/view/4563/3920 22.Ministério da Saúde (Br). Secretaria de Vigilância em Saúde /Departamento de DST, Aids e Hepatites Virais. Hepatites virais no Brasil: situação, ações e agenda. Brasília (DF): Ministério da Saúde; 2011.

23.Shin BM, Yoo HM, Lee AS, Park SK. Seroprevalence of Hepatitis B virus among health care workers in Korea. J Korean Med Sci, 2006; 21: 58-72 [cited in 2014 Nov 08] Available in: http://www.ncbi.nlm.nih.gov/pmc/articles/ PMC2733980/

24.Silva PA, Fiaccadori FS, Borges AMT, Silva SA, Daher RR, Martins RMB. Seroprevalence of hepatitis $\mathrm{B}$ virus infection and seroconvertion to anti-HBsAg in laboratory staff in Goiânia, Goiás. Rev Soc Bras Med Trop. 2005: 38(6): 153-6.

25.Ciorlia LAS, Zanetta DM. Significado epidemiológico dos acidentes de trabalho com material biológico: hepatites B e C em profissionais da saúde. Rev. Bras. Med. Trab.2004; 2: 191-9.

26.Nazar AN. Bastos AP, Pittella AM, Matos HJ. Análise da soropositividade do Anti-HBs em profissionais de saúde. Cad Saúde Colet. 2008; 16: 421-36

27.Faraht C, Carvalho ES, Weckz L, Carvalho LHFR, Succi LCM. Imunizações: fundamentos e prática. São Paulo: Atheneu; 2000.

28. Toledo AD, Oliveira AC. Situação vacinal e sorológica para hepatite $b$ entre trabalhadores de uma unidade de emergência. Rev enferm UERJ. 2008; 16: 95-100. 